\title{
Case Study: Blended Learning Approach in Pool Lifeguard Training
}

\author{
https://doi.org/10.3991/ijac.v13i3.17511 \\ Shaun Jackson $(\bowtie)$ \\ Royal Life Saving Society - Australia, Sydney, Australia \\ sjacksonerlssa.org.au
}

\begin{abstract}
The Pool Lifeguard Skill Set (SISSS00111), recognized across Australia, is targeted at those that wish to be professionally certified as a Pool Lifeguard in Australia. Incorporating risk management, supervision, communication, aquatic rescue and emergency care training, the audience covers a broad demographic in city and regional locations. The course has traditionally been taught through pre-course workbooks, reference to a manual, and face-to-face training sessions. While this traditional approach has been effective, Royal Life Saving wished to offer a more engaging and memorable pre-course delivery method. The goal was to enable students to arrive at the face-to-face training with a stronger, more embedded knowledge of key concepts and principles and allow for more face-to-face training time to be devoted to becoming proficient in the role's important practical skills, such as aquatic rescue, first aid and advanced CPR. Hence, a project to develop an e-learning blended delivery solution was undertaken. The intention was that the online component of the course be completed prior to the face-to-face training and that it should augment the lifeguarding manual. It was also important that it is highly learner-centred, requiring learners to interact with the screens in order to access the key content and that it is a visually pleasing product with a quality aesthetic design.
\end{abstract}

Keywords - Blended learning, e-learning, elearning

\section{Introduction}

Pool Lifeguards prevent dangerous incidents and save lives at aquatic facilities. Royal Life Saving had been an industry leader in pool lifeguard training since its very beginning and currently delivers the course in each state and territory in Australia. The RLS network identified a growing need to update and revitalise the pool lifeguard course. This need was generated from technical changes to the practice of lifeguarding but also internally in order for RLS to consolidate its position as an industry leader and to make better use of digital learning technologies, and thereby offer a more familiar and memorable learning experience to its younger audience. At the same time, the RLS network of Registered Training Organizations (RTOs) had made a series of other information communication technology changes, e.g., a new training management 
system, that would better enable it to offer a high quality, nationally consistent elearning product. It was now time to work collaboratively to create the product.

\section{Learning Design}

The e-learning Pool Lifeguard Suite is made up of seven 15-20 minute modules that focus on providing an engaging learning experience and giving insight into the practical application of the fundamental job requirements, skills and practices of a pool lifeguard.

The design was based on the following considerations:

- A predominately young target audience diverse in education and knowledge with a high exposure to online environments.

- A universal instructional method to accommodate a range of learning styles and learner backgrounds.

- Use of an experienced lifeguard as a narrator, role-model and proxy throughout the modules.

- Presentation of new information that compels learners to call on existing knowledge and skills, e.g., critical thinking and reasoning, problem solving, etc., in order to make decisions - answer a question - and then learn more through immediate feedback. The series of images below shows how this approach is incorporated into the course through a Hint or Try task.

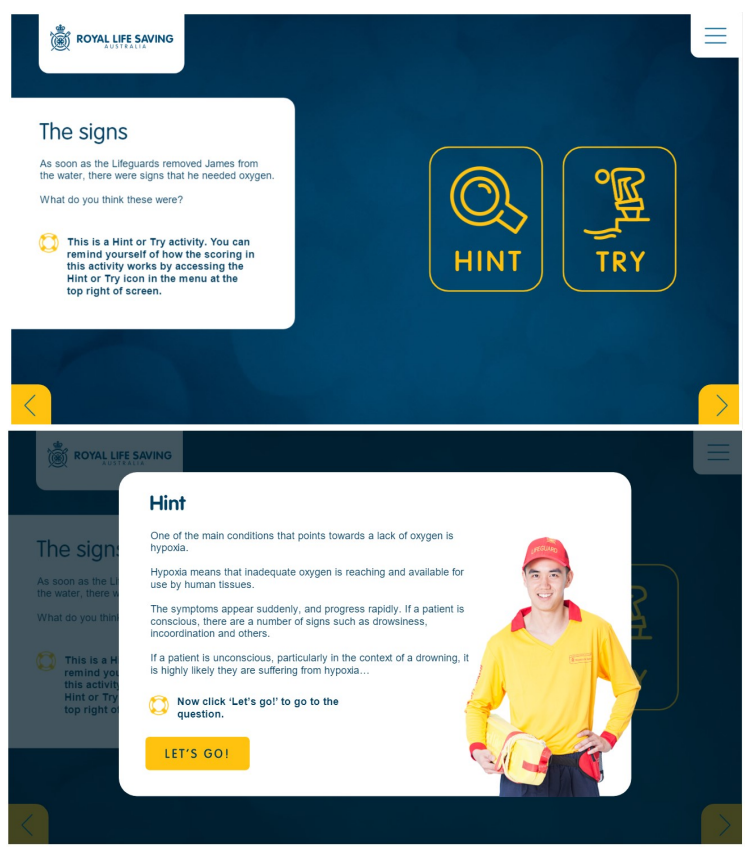




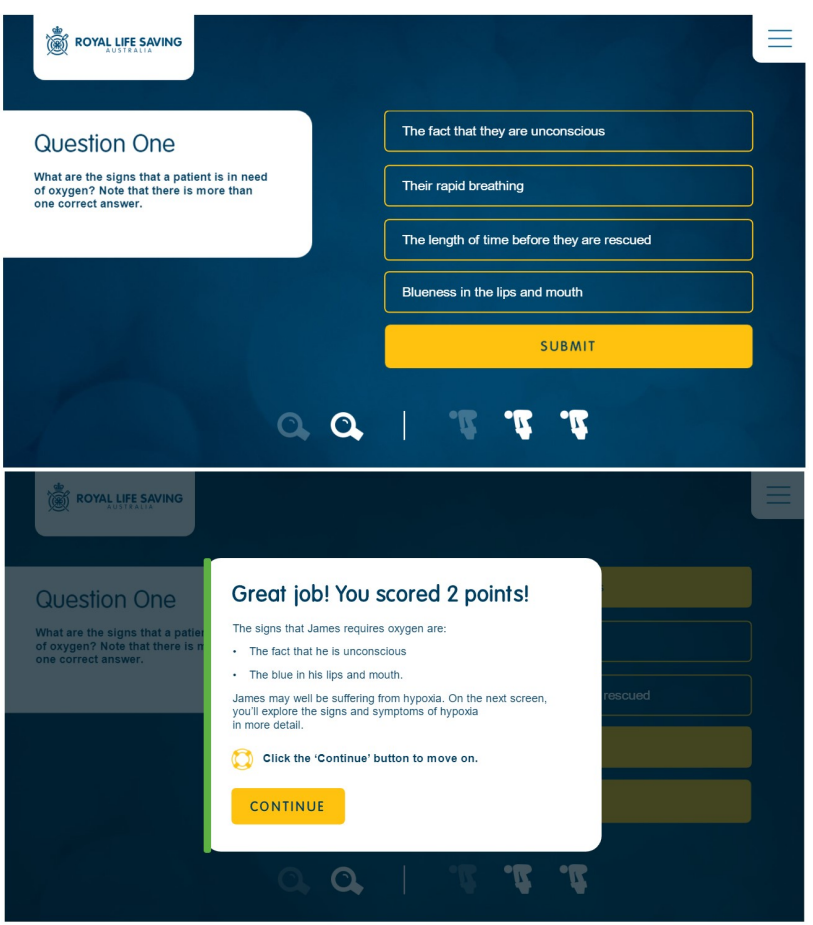

- Use of authentic and realistic scenarios as a springboard into key content. The series of images below shows how a typical scenario at an aquatic facility is used to present content to the learner on the principles of rescue, i.e., Awareness, Assessment, Action, After Care.

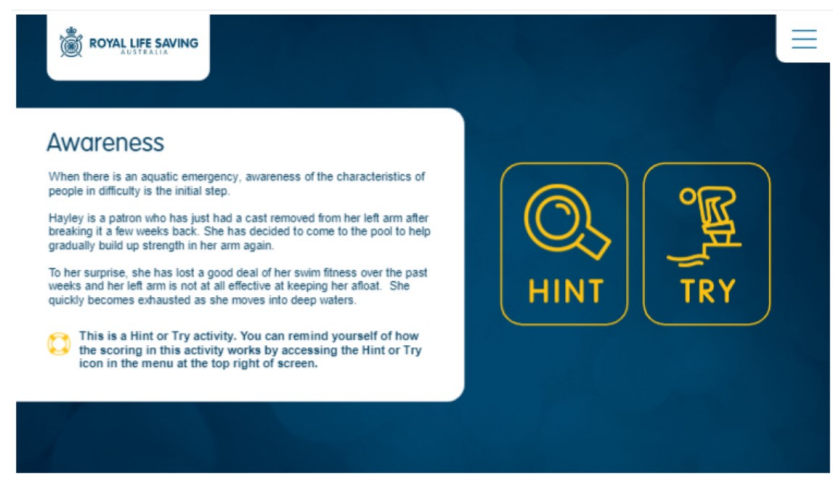



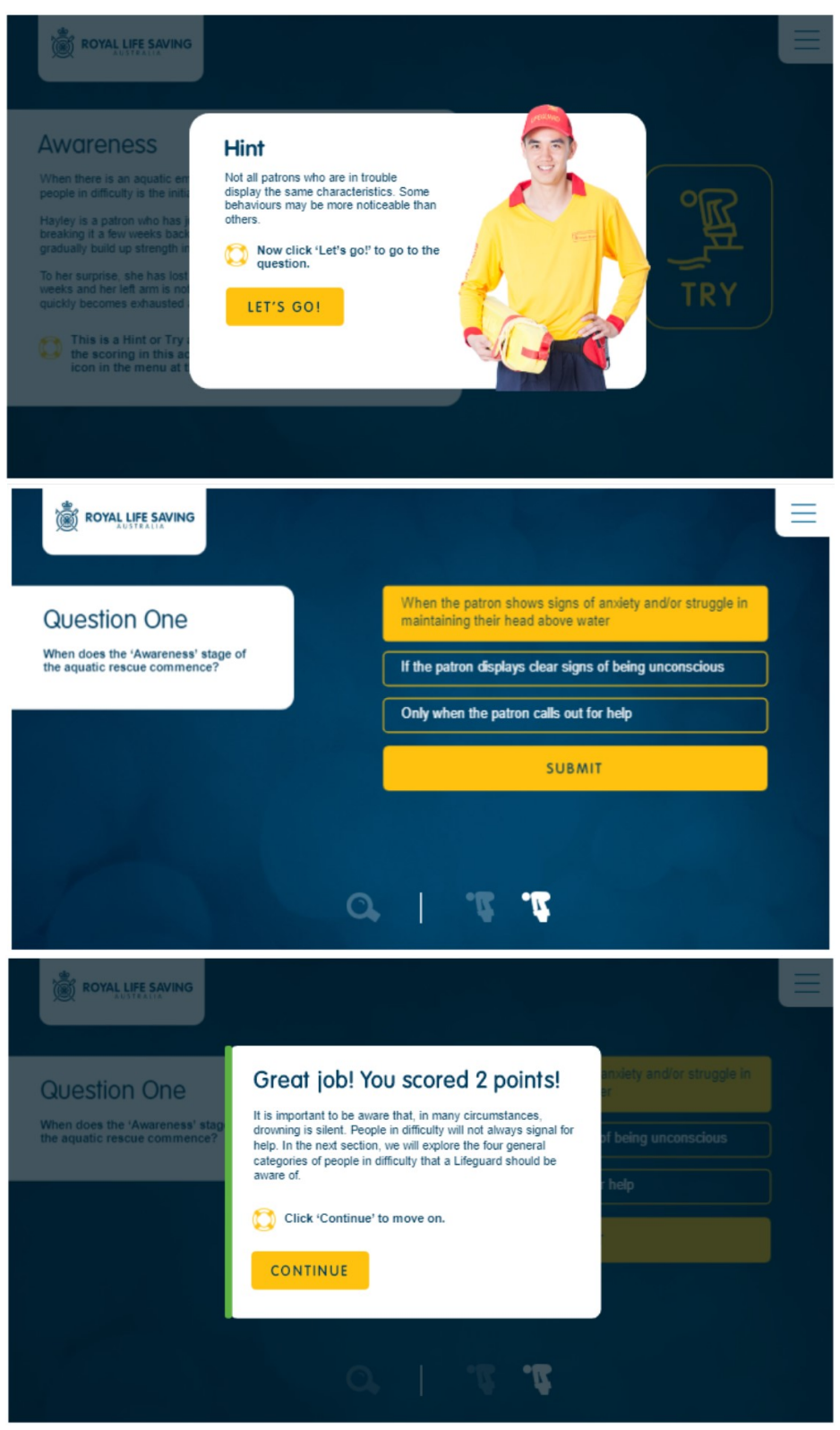


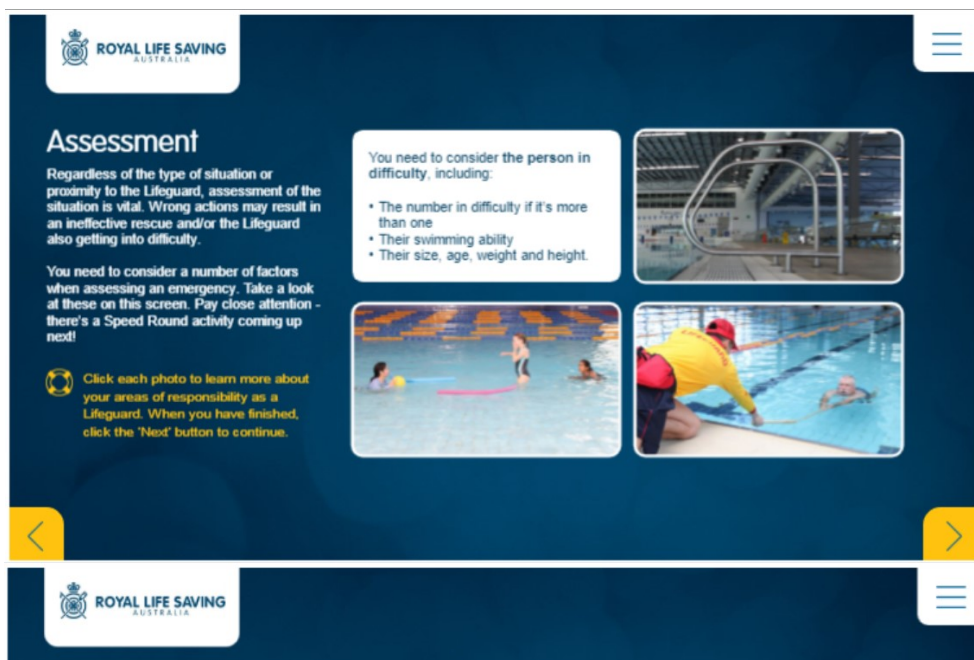

\section{Action}

II's now time to take action. Due to Hayley's echaustion and injured arm, she is unable to
keep hersetf alloat The Uthend keep herself alloat. The Uliteguard has
siluation and identilited the following:

- Hayley is strugghing to keep her head above water and is - Hayley is currentily in the midde of the pod in water that is tor deep lor her to to stand in
- Hayliey is in her late teens and is slightly smaller than the - Neadty resources include a recreational pool noodle and a
rescoue tuthe

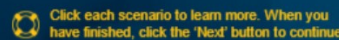

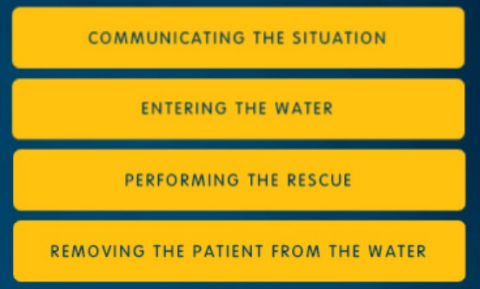

$<$
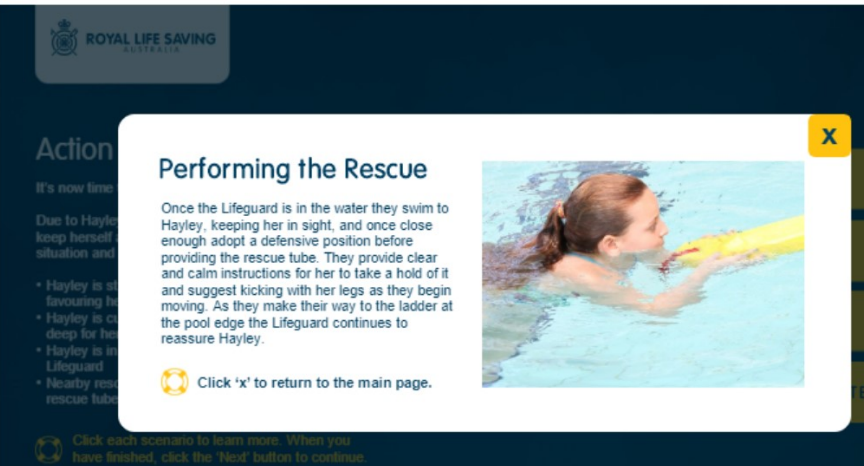

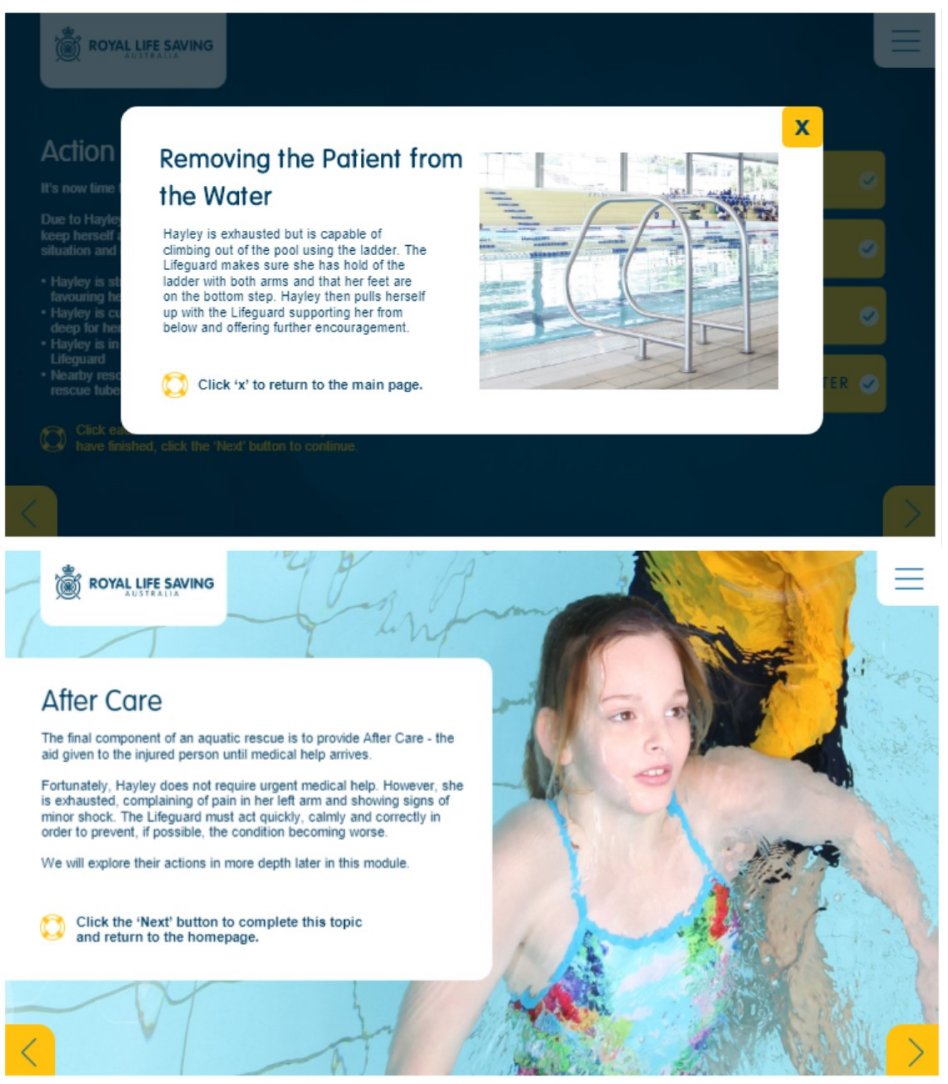

- Use of a capstone video scenario, i.e., a filmed rescue scene, to introduce advanced resuscitation skills and reinforce concepts covered in earlier modules (supervision, communication, risk management, rescue) in a highly memorable format.

- Use of gamification to increase engagement and facilitate retention of key themes and specific content.

- Application and reinforcement of key concepts (such as maintaining technical accuracy under time pressure, following policy and procedures, communicating and collaborating effectively and making informed decisions) are reflected in the design and requirements of specific tasks, e.g., the hint or try and speed tests. The series of images below shows how the design of a specific task, i.e., a speed test gamified task is used to increase engagement and facilitate retention of key concepts. 


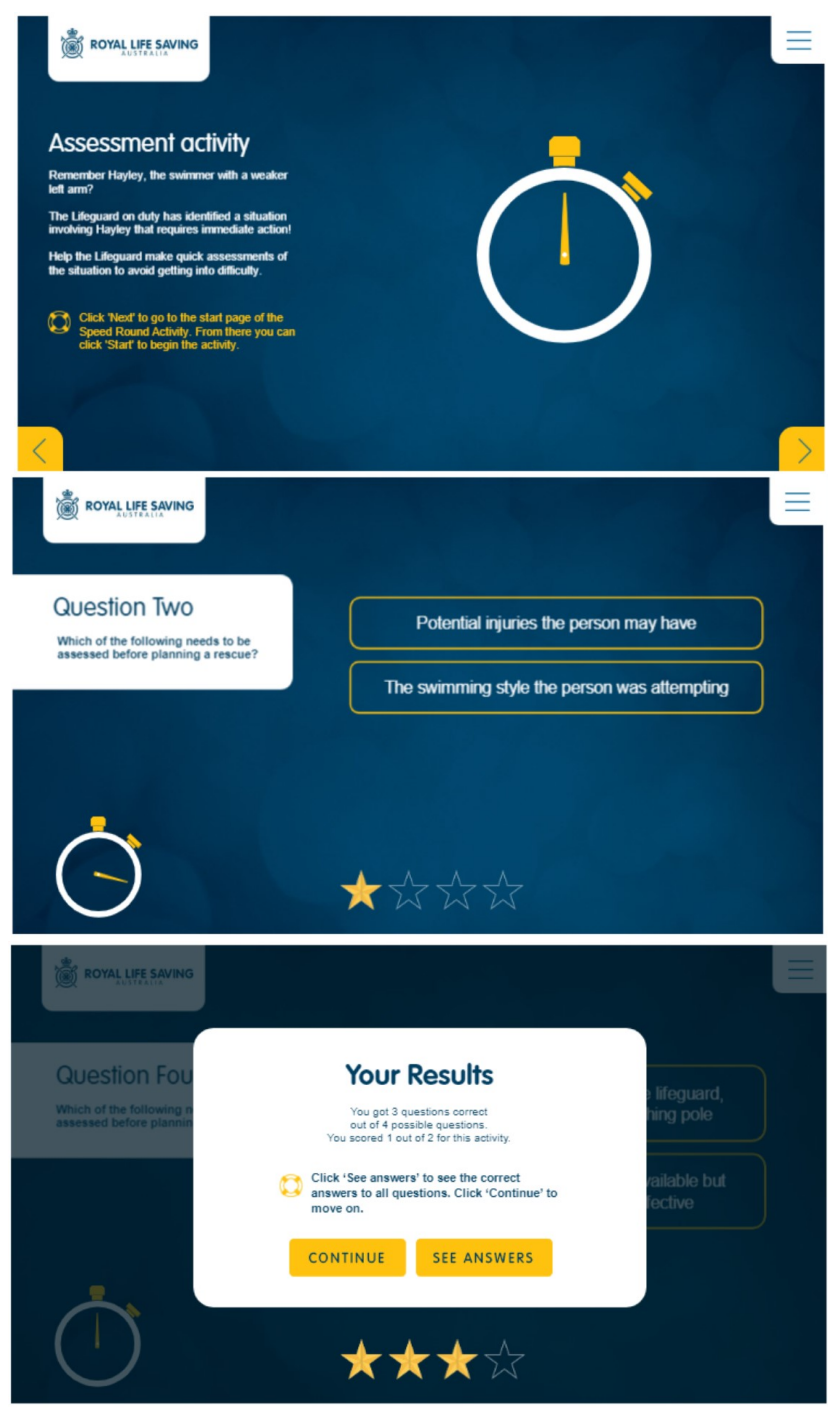

- Learners will have approximately two days of face-to-face training, plus be able to refer to a lifeguarding manual, to consolidate and expand their learning. Hence the role of the pre-course e-learning was to introduce key concepts and content and require learners to understand, consider and apply these in the context of realistic settings and scenarios.

The learner is introduced to Lochie, an experienced and high-achieving lifeguard, who provides a sense of wisdom and authority on lifeguarding matters. Lochie is accessible throughout the modules for practical help as well as often performing the role of intermediary into important content, usually through personal stories or anecdotes. 


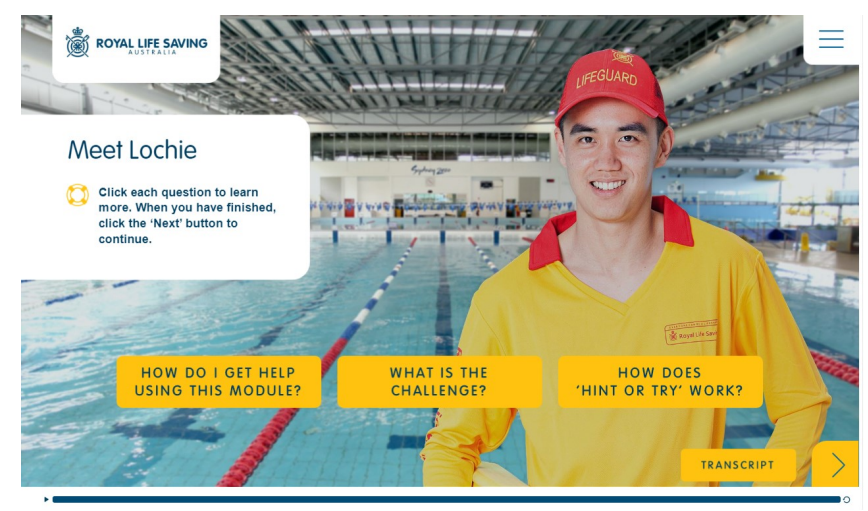

Fig. 1. Meet Lochie

The modules are underpinned by a gamification framework that forms a central component of the learning design. The use of hint or try activities and speed tests reinforces key concepts around the need to balance informed decision making through concepts such as effective questioning and active listening, with having a strong grasp of basic knowledge and techniques in order to act quickly and confidently, e.g., use of hand signals. Through the gamified activities the learner strives for the best possible merit award at the end of each module: still preparing, prepared, well prepared.

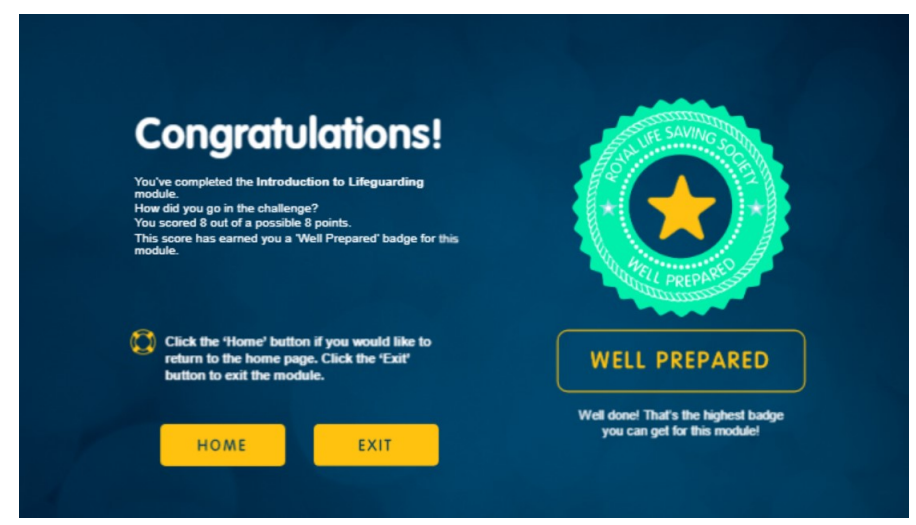

Fig. 2. Merit badge screen

In line with the above points, the learning design underpinning the course is process and behaviour-focused - the model allows for theory and principles to be presented, i.e., the what, in authentic contexts that demonstrate why it is important and the bestpractice response, i.e., the how. 


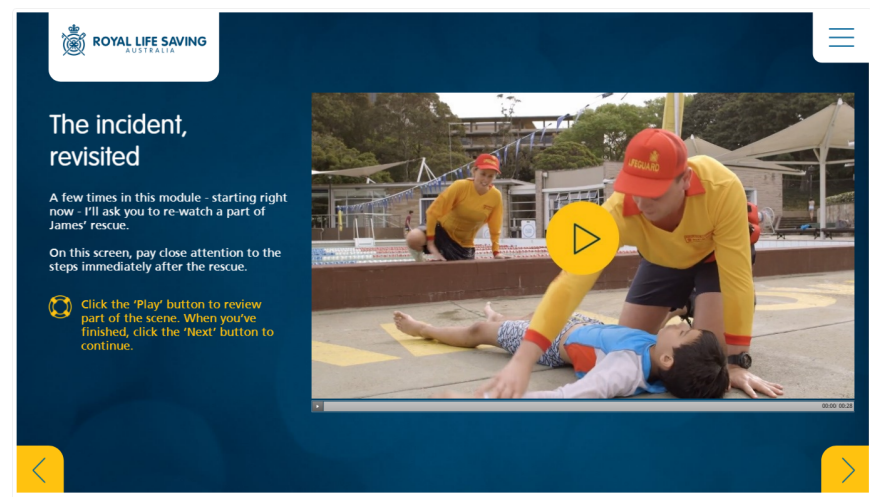

Fig. 3. The rescue and resuscitation scenario

\section{$3 \quad$ Program Delivery}

The seven pool lifeguard modules sit as SCORM files within the various RLS training management systems (TMS) and upon confirmation of enrollment participants receive an email with a link to the e-learning modules within their RLS learner portal. This email explains that completion of the seven modules (plus an assessment module that was created after the initial project) is necessary prior to attending the face-to-face training. The design of the modules allows RLS to decide which modules are applicable for an individual or particular cohort, and it may be that only certain modules are provided, e.g., for lifeguards completing a reaccreditation course they may complete the rescue and resuscitation modules only. That being said, for those accessing the course for the first time it is strongly encouraged that they complete module one first and participants new to pool lifeguarding will typically complete all seven modules. Within each module there are a number of topics, which cover one to three learning points at most.

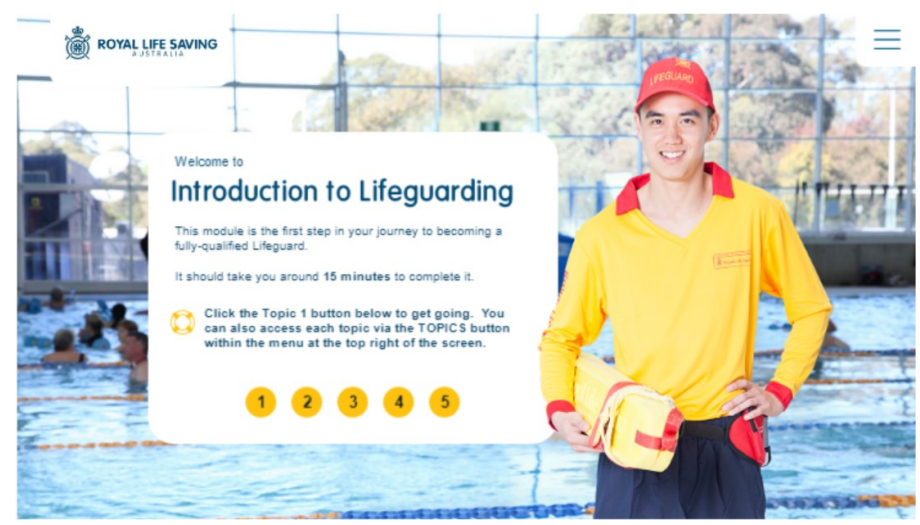

Fig. 4. Landing page 
As mentioned, the online modules form a pre-course component in preparation for two days of face-to-face training. Learner progress and completion of each module is monitored through the Organisation's TMS and reminder emails are sent at specific points leading up to the face-to-face component. Monitoring has revealed that students typically engage with the modules just after enrollment and then just prior to commencing the face-to-face component. The seven modules are usually completed in two or three blocks. The assessment module is typically completed in the one to two days prior to the face-to-face training. Ideally learners would complete the modules in a more consistent, regular pattern and leave more time to complete the assessment module, as this can be quite challenging and typically requires learners to revisit the modules and/or refer to the Manual.

\section{$4 \quad$ Results}

Feedback from RLS trainers is that learners are coming to the face-to-face training with a broader knowledge set and greater command of key concepts. This is borne out in the fact that they are more cognisant of key concepts, are asking more focused and technical questions and are regularly referring to the online modules to support their queries and observations. RLS is closely connected to the aquatic industry and in regular contact with aquatic facility managers and it has been pleasing to receive feedback that during interviews, induction and early work stages new lifeguards are generally demonstrating greater knowledge of the fundamental components of the role and also improved technical skills around supervision, rescue and resuscitation. This aligns with feedback from trainers that they are able to devote more face-to-face training to developing these essential skills.

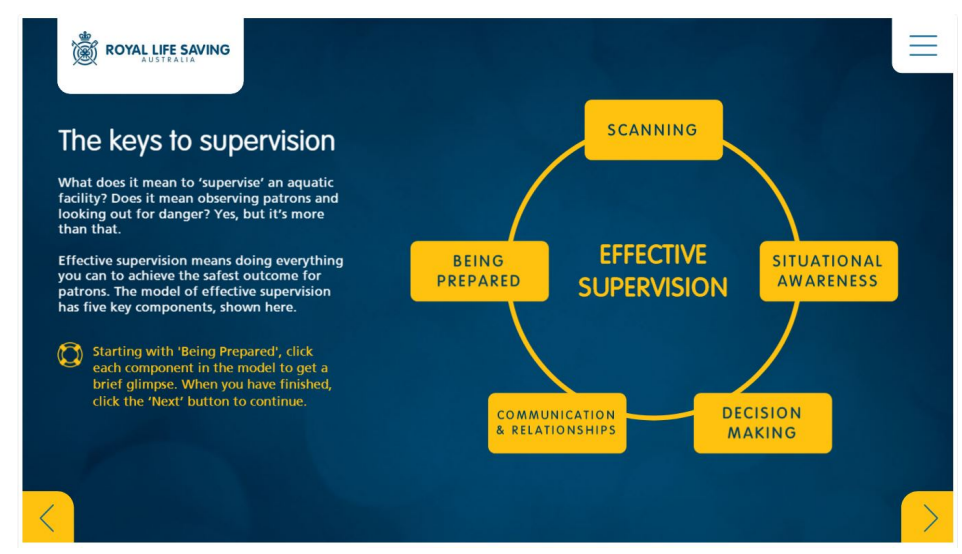

Fig. 5. Sample content screen - Elements of supervision

Overall, the blended learning approach has been very successful. The overall adoption and positive uptake have resulted in 2000 participants undertaking the course within NSW, TAS, WA and ACT since its release - significant growth for these member 
organisations. The trainers are pleased to have more time to devote to practical application of the skills and running scenarios and the automation around enrollment and completion, when compared to using pre-course workbooks, has resulted in reduced costs and administration work for back office staff.

Learner feedback, gathered from end of course surveys and anecdotally throughout the face-to-face training, has also been overwhelmingly positive. Learners are particularly positive about the use of the filmed rescue scenario. Comments centre on how this gives them a powerful insight into what the role may involve and how it reinforces - and makes real - many of the key concepts covered in the earlier modules. In line with this, learners have queried why there is not more content on rescue techniques in the e-learning modules. Given this is covered in the face-to-face training it was not included in the original version of the e-learning suite, but work is underway to add this content to an updated version.

\section{Author}

Shaun Jackson is the National Manager - Training and Workforce Development at Royal Life Saving Society - Australia. Shaun has managed several Registered Training Organisations and English language colleges over more than 20 years in the vocational education and training industry. Shaun started out as English language teacher while completing a Bachelor of Arts degree at University of Sydney (Philosophy) and the International Diploma in Language Teaching Management at University of Queensland. Shaun has lived, studied language and worked in workforce training and English language teaching in London, England, Bologna, Italy, and Berlin, Germany. He now lives with his young family in Sydney, Australia. Email: sjackson@rlssa.org.au

Article submitted 2020-08-03. Resubmitted 2020-08-25. Final acceptance 2020-08-25. Final version published as submitted by the authors. 